\title{
Chancen der Digitalisierung - Transaktionskosten im traditionellen Geschäft senken, neue Interaktionen verstärken
}

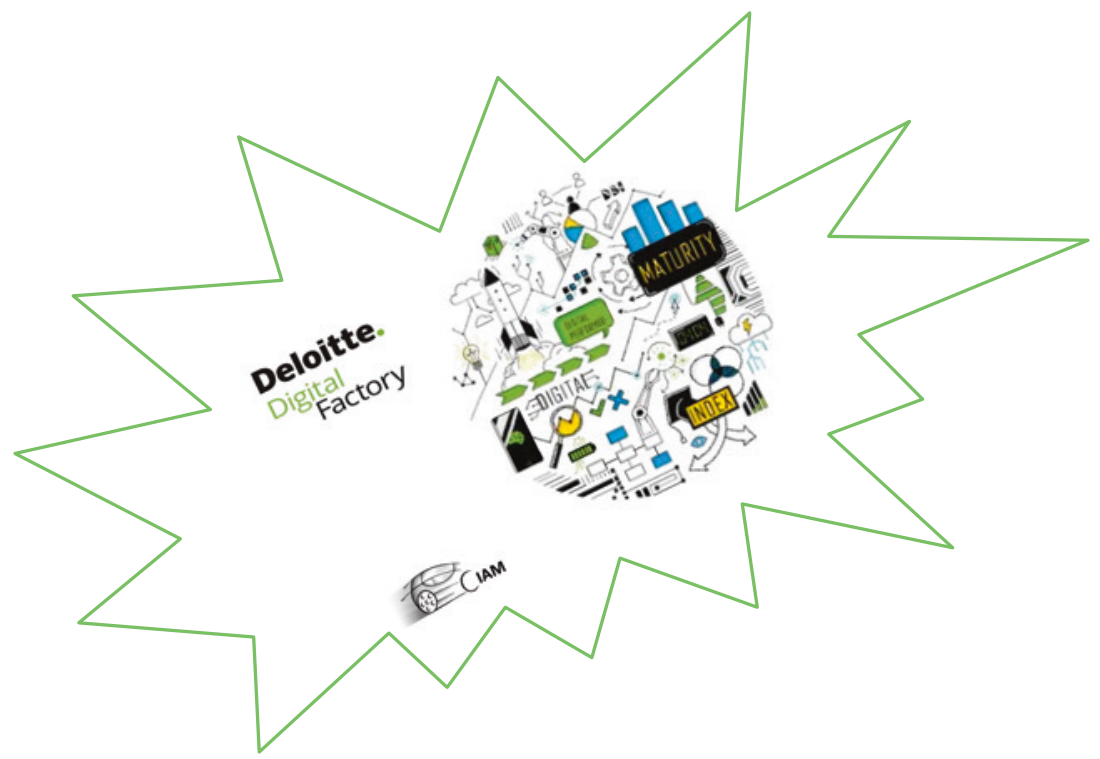

Elektronisches Zusatzmaterial Die elektronische Version dieses Kapitels enthält Zusatzmaterial, das berechtigten Benutzern zur Verfügung steht. https://doi. org/10.1007/978-3-658-31456-9_2. Die Videos lassen sich mit Hilfe der SN More Media App abspielen, wenn Sie die gekennzeichneten Abbildungen mit der App scannen. 
Wir wollen in diesem Kapitel begründen, welche Chancen die Digitalisierung dem Management bietet:

1. Gemäß der Transaktionskostentheorie ermöglicht Digitalisierung eine Standardisierung von Schnittstellen und damit eine Verringerung von Interdependenzen entlang der Wertkette im traditionellen Geschäft und damit

* eine Verringerung der (Transaktions-)Kosten

(1) der internen Koordination durch bessere Standardisierung unternehmensinterner Schnittstellen, wodurch z. B. schneller mehr Varianten profitabel angeboten werden können,

(2) der externen Koordination durch Standardisierung der Schnittstellen zu Lieferanten, wodurch eine Auslagerung von eigener Wertschöpfung auf kostengünstigere Lieferanten erleichtert wird sowie

(3) der externen Koordination durch Standardisierung der Schnittstellen zu Kooperationspartnern, um durch gemeinsame Wertschöpfung Synergien zu erzielen und

* dadurch auch

(4) eine Verringerung von Zielkonflikten zwischen Effizienz und Flexibilität, wodurch Handlungsspielräume für eine bessere Ausrichtung im Wettbewerb geschaffen werden.

2. Gemäß den Erklärungen der Interaktion ermöglicht Digitalisierung zudem eine bessere Zusammenarbeit mehrerer externer Akteure auf technischen Plattformen als Voraussetzung für innovative Kundenlösungen und Geschäftsmodelle in neuartigen Netzwerken (Ecosystems).

Damit schafft die Digitalisierung dem Management Handlungsspielräume und ermöglicht Gewinnpotenziale.

\subsection{Verringerung der Transaktionskosten im traditionellen Geschäft entlang der Wertkette durch Digitalisierung}

Die Digitalisierung ermöglicht eine Standardisierung von Schnittstellen. Dadurch verringert sie Interdependenzen entlang der Wertkette, z. B. zwischen und innerhalb von Fertigung, Logistik und Vertrieb. Damit sinken auch die 
Transaktionskosten ${ }^{1}$ im bisherigen, traditionellen Geschäft, d. h. die Kosten der Abstimmung - unternehmensintern, aber auch mit externen Partnern (vgl. Abb. 2.1). Dies lässt sich mithilfe der Transaktionskostentheorie begründen. ${ }^{2}$

Die Wert(schöpfungs)kette in einem bestehenden Markt wurde durch den Harvard-Ökonom Michael Porter bekannt und wird als System interdependenter bzw. verknüpfter Aktivitäten verstanden, durch deren adäquate Koordination sich ökonomische Gewinne erzielen lassen. ${ }^{3}$ Gemäß der entscheidungsorientierten Organisationstheorie ${ }^{4}$ bestehen Interdependenzen entlang der Wertkette dann, wenn die Entscheidungen einer Unternehmenseinheit die Entscheidungen einer anderen Einheit ,zielrelevant verändern“5 und die Lösung komplexer Entscheidungen in arbeitsteiligen Entscheidungsprozessen begrenzen. Nach ihrer Stärke kann zwischen restriktiven, sequenziellen und „gepoolten“ Interdependenzen unterschieden werden, die den Verknüpfungen zwischen primären, unterstützenden und Infrastrukturaktivitäten in der Wertkette von Porter (vgl. ebenfalls Abb. 2.1) entsprechen.

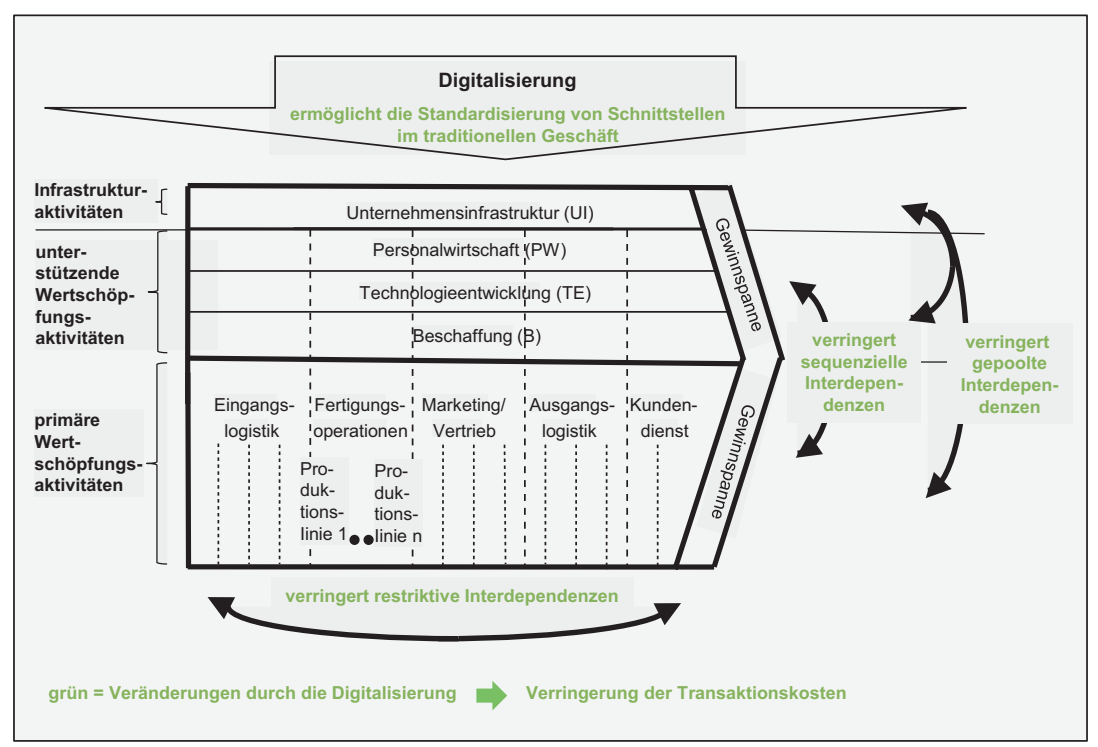

Abb. 2.1 Verringerung von Transaktionskosten im traditionellen Geschäft durch verringerte Interdependenzen entlang der Wertkette durch die Digitalisierung. (Quelle: Proff, 2019 bezogen auf Porter, 1985) 
Bei restriktiven Interdependenzbeziehungen zwischen Entscheidungseinheiten fehlen Puffer, die Freiheitsgrade sind damit sehr gering. Die primären wertschöpfenden Entscheidungseinheiten Fertigung, Logistik und Vertrieb stehen in einer solchen restriktiven Interdependenzbeziehung und müssen deshalb stark aufeinander abgestimmt werden. Werden z. B. die Entscheidungen der Fertigungseinheit über die Höhe und die zeitliche Verteilung des Produktionsprogramms optimiert, dann muss die Logistikeinheit genau auf dieses Produktionsprogramm abgestimmt werden und die Vertriebseinheiten können auf veränderte Nachfragebedingungen kaum mehr flexibel reagieren.

Sequenzielle Interdependenzbeziehungen bestehen, wenn eine Entscheidungseinheit in einer Handlungssequenz mit anderen Entscheidungseinheiten steht. ${ }^{6}$ Nach Porter stehen die unterstützenden Aktivitäten Beschaffung, Personalwirtschaft und Technologieentwicklung bzw. FuE mit den primären Aktivitäten in einer solchen Beziehung, da sie von diesen weitgehend unabhängig sind. ${ }^{7}$ Die Vorteile eines günstigen Zugangs zu Rohstoffen lassen sich z. B. weitgehend unabhängig von Fertigungsoperationen anstreben. Verzögert sich jedoch die Auslieferung von Ressourcen durch die Beschaffungseinheit, so beeinflusst dies die Fertigungseinheit negativ.

„Gepoolte“ Interdependenzbeziehungen bestehen, wenn eine Entscheidungseinheit unabhängig von den Entscheidungen der anderen Einheiten agieren kann. Porter spricht von Einheiten der „Unternehmensinfrastruktur“ und fasst darunter Managementaufgaben wie Finanzwirtschaft, Rechnungswesen und Öffentlichkeitarbeit. Die Infrastrukturaktivitäten unterstützen die gesamte Wertkette. $^{8}$

Die Digitalisierung ermöglicht durch die Standardisierung von Schnittstellen zwischen allen Aktivitäten entlang der Wertkette im traditionellen Geschäft eine Verringerung von restriktiven, sequenziellen und auch gepoolten Interdependenzen, ${ }^{9}$ wodurch sich die Wertschöpfungsaktivitäten entkoppeln lassen. Damit können die Entscheidungseinheiten viel selbstständiger und ,,bis zu einem gewissen Grad getrennt voneinander" Entscheidungen treffen ${ }^{10}$ (Abb. 2.1).

Mit sinkenden Interdependenzen entlang der Wertkette verringert die Digitalisierung auch Transaktionskosten im traditionellen Geschäft (vgl. ebenfalls Abb. 2.1):

1. Transaktionskosten der internen Koordination aufgrund besserer Standardisierung von unternehmensinternen Schnittstellen, ${ }^{11}$ was schnell viele Varianten erlaubt, 
2. Transaktionskosten der externen Koordination durch bessere Standardisierung von Schnittstellen zu den Lieferanten, was eine Auslagerung auf Lieferanten erleichtert,

3. Transaktionskosten der externen Koordination durch bessere Standardisierung der Schnittstellen zu Kooperationspartnern, was Synergien durch gemeinsame Wertschöpfung ermöglicht und

4. dadurch den Zielkonflikt zwischen Effizienz und Flexibilität lösen hilft, was Handlungsspielräume für eine bessere Positionierung im Wettbewerb schafft.

Die Digitalisierung wirkt aber auch innerhalb der von Porter recht grob unterschiedenen Wertschöpfungsaktivitäten, z. B. entlang einzelner Produktionslinien, die bei globaler Betrachtung sehr komplex sein können (vgl. ebenfalls Abb. 2.1).

Die Verringerung der internen und externen Transaktionskosten zwischen und innerhalb der Wertschöpfungsaktivitäten im traditionellen Geschäft durch die Digitalisierung lässt sich am Beispiel einer Produktionslinie in der Deloitte Digital Factory in Düsseldorf zeigen, die nach einem Kundenauftrag durchlaufen wird und auf die Logistik- und Vertriebsaktivitäten folgen. (vgl. den folgenden Kasten):

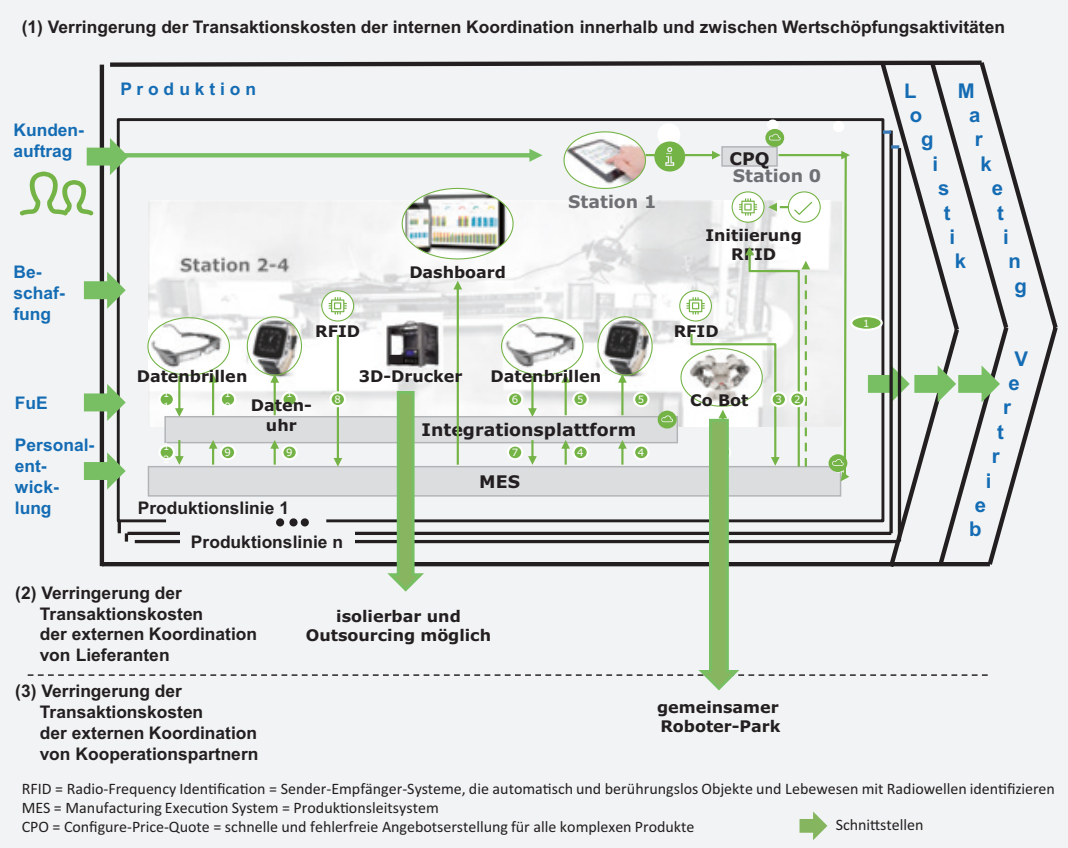


Die Produktion zwischen Kundenauftrag und Logistik an den Kunden (und damit zu Marketing und Vertrieb) wird im traditionellen Geschäft nach Lean-Kriterien optimiert. Sie ist (sind) aber vollkommen analog.

Im Zuge der Digitalisierung erfolgt über verschiedene Zwischenschritte eine Umrüstung der Produktionslinie mithilfe eines verknüpfenden Produktionsleitsystems (Manufacturing Execution System, MES) als gemeinsamer Datenplattform. Zudem werden über eine Integrationsplattform z. B. Datenbrillen, RFID-(Radio-Frequency-Identification-)Chips, die Objekte mit Radiowellen identifizieren, Digitaluhren und digitale (KPI-)Dashboards, die wichtige Unternehmenskennzahlen in Echtzeit visualisieren und ihre Zielwirkung zeigen, integriert. Dies führt zu standardisierten internen Schnittstellen zwischen den einzelnen Elementen der Produktionslinie. Zudem können interne Schnittstellen zu anderen Wertschöpfungsaktivitäten standardisiert werden, z. B. ausgehend von FuE, Beschaffung und Personalentwicklung sowie ausgerichtet auf Logistik und Marketing/Vertrieb (vgl. ebenfalls den Kasten). Die Arbeiten an der Produktionslinie der Deloitte Digital Factory in Düsseldorf haben gezeigt, dass sich durch die Digitalisierung fast die Hälfte der unproduktiven Wartezeit innerhalb einer Produktionslinie und etwa 10 Prozent der reinen Produktionszeit einsparen lassen (vgl. Abb. 2.2(1)). Diese Zeitersparnis, die durch die Standardisierung der Schnittstellen zu anderen Wertschöpfungsaktivitäten noch vergrößert werden kann, kann in Kostensenkungen oder in zusätzliche Produktionsvolumina/varianten umgesetzt werden.

Im Zuge der Digitalisierung kann das Produktionsleitsystem (MES) der einzelnen Produktionslinien auch für Externe geöffnet werden. Die klaren Schnittstellen der Produktionsmodule und die im gesamten Prozess gewonnene Zeit ${ }^{12}$ erlauben dann eine Auslagerung (,outsourcing“) auf externe Lieferanten. So besteht z. B. die Möglichkeit, den 3D-Drucker, der hohe Investitionskosten verursacht, aus den eigenen Fertigungsprozessen auszugliedern und 3D-Druckteile von einem Lieferanten produzieren zu lassen, der alle relevanten Daten rechtzeitig erhält. Eine Deloitte-Befragung von 155 Unternehmen des verarbeitenden Gewerbes zu ihren Produktionsstrategien ${ }^{13}$ zeigt, dass eine höhere Digitalisierungsaffinität mit einer höheren Auslagerung einhergeht (vgl. Abb. 2.2(2)).

Durch Öffnung des Produktionsleitsystems für Externe wird auch eine gemeinsame Wertschöpfung mit Kooperationspartnern möglich. So besteht z. B. die Möglichkeit, gemeinsam Roboter-Parks zu betreiben, in die z. B. Montageroboter - sog. kollaborative Roboter (CoBots) - ausgelagert werden, d. h. Industrieroboter, die mit Menschen zusammenarbeiten (vgl. ebenfalls den Kasten). In der Deloitte-Befragung zu den Auswirkungen der Digitalisierung auf die Produktionsstruktur von Unternehmen des verarbeitenden Sektors in 
Deutschland zeigt sich, dass Unternehmen mit einer hohen Digitalisierungsaffinität deutlich häufiger Vermögenswerte (,shared assets“) teilen und damit kooperieren als Unternehmen mit einer geringen Digitalisierungsaffinität (vgl. Abb. 2.2(3)).

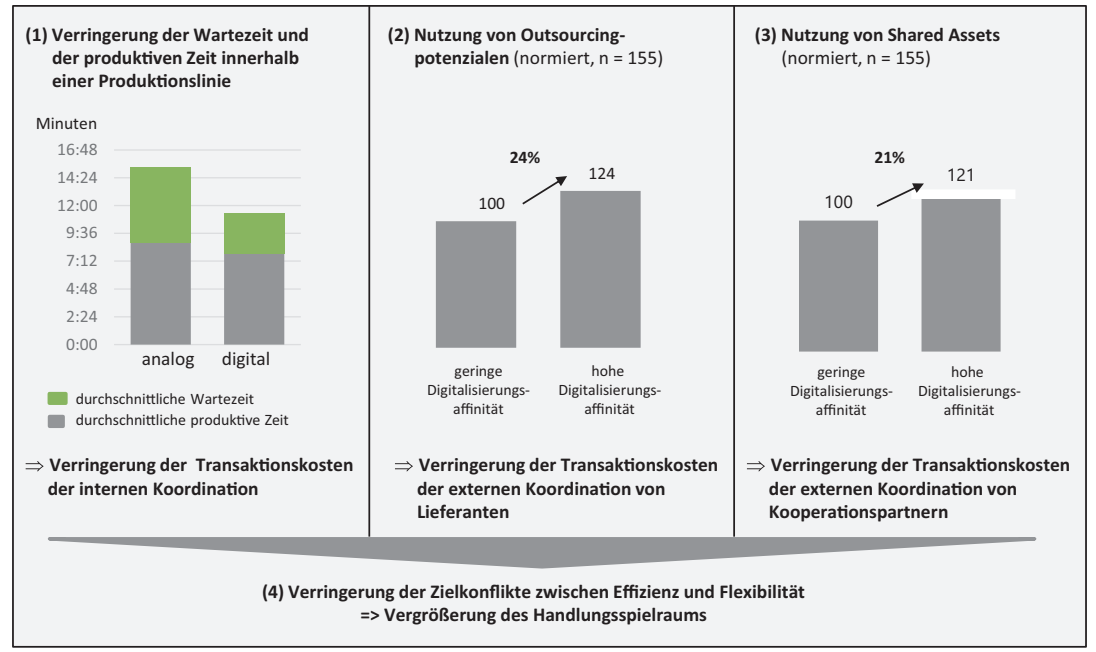

Abb. 2.2 Wirkung der Digitalisierung auf die Nutzung externer Produktionsnetzwerke. (Quelle: Deloitte 2020a)

Die Verringerung der ex- und internen Transaktionskosten durch die Digitalisierung im traditionellen Geschäft führt schließlich zu einer Verringerung der Zielkonflikte zwischen Effizienz und Flexibilität und vergrößert damit den Handlungsspielraum der Wettbewerber (vgl. ebenfalls Abb. 2.2(4)). Hier zeigt sich, warum die Digitalisierung häufig als Ermöglicher von Chancen (,enabler" ${ }^{614}$ bezeichnet wird.

Die im Kasten beispielhaft dargestellten und durch Hinweise auf Erfolgswirkungen in Abb. 2.2 gestützten Effekte der Digitalisierung lassen sich auch erklären, was im Folgenden mithilfe der Transaktionskostentheorie kurz geschieht.

\section{(1) Verringerung der Transaktionskosten der internen Koordination im traditionellen Geschäft durch die Digitalisierung}

Die Digitalisierung ermöglicht eine bessere Standardisierung unternehmensinterner Schnittstellen zwischen den einzelnen Elementen der Wertkette im 
bisherigen, d. h. traditionellen Geschäft. Dies hat Einfluss auf die Transaktionskosten der internen Koordination, also auf die Kosten des Austauschs von Vermögenswerten - Kapital wie Gütern - in der Hierarchie des Unternehmens (,make“). Gemeint sind die Kosten der Anbahnung, Vereinbarung und Abwicklung sowie nachträgliche Kontrollen und Anpassungen von Transaktionen im traditionellen Geschäft. Diese Transaktionskosten erhöhen sich grundsätzlich mit steigender strategischer Bedeutung bzw. mit steigender (Faktor-)Spezifität eines Vermögenswertes, d. h. mit spezifischer Ausrichtung auf eine bestimmte Verwendung, was eine profitable Wiederverwertbarkeit für andere Anwendungen beeinträchtigt. Die Transaktionskosten wachsen aber auch mit steigender Unsicherheit - sowohl mit Ungewissheit über künftige Umweltzustände als auch mit Verhaltensunsicherheit ${ }^{15}$ (vgl. Abb. 2.3).



Abb. 2.3 Verringerung der Transaktionskosten der internen Koordination im traditionellen Geschäft durch die Digitalisierung. (Quelle: nach Picot u. a. 2015) 
Die Transaktionskosten in Unternehmen mit oft hierarchischeren Entscheidungsprozessen sind bei geringer Faktorspezifität (z. B. einmaligen Transaktionen bei Standardteilen wie z. B. Schrauben) meist höher als bei einem Fremdbezug bei Lieferanten am Markt (,buy“). Die Transaktionskosten mit Lieferanten am Markt steigen aber mit steigender Faktorspezifität (mit stärkerem Austausch bzw. Partnerschaften bei der Beschaffung großer Module) schneller als im Unternehmen (vgl. ebenfalls Abb. 2.3).

Durch die Digitalisierung können nicht nur Vor- und Zwischenprodukte, sondern auch Informationen schneller und einfacher innerhalb eines Unternehmens transferiert werden. ${ }^{16}$ Damit reduzieren sich die Transaktionskosten der internen Koordination im traditionellen Geschäft. Das Potenzial dieser Kosteneinsparung kann durch verstärkte Eigenfertigung (,make“) im Unternehmen realisiert werden (Abb. 2.3) und entweder für zusätzliche Wertschöpfung (Insourcing oder Erhöhung der Variantenvielfalt) genutzt oder als verringerte Kosten einbehalten werden.

\section{(2) Verringerung der Transaktionskosten der externen Koordination von Lieferanten im traditionellen Geschäft durch Digitalisierung}

Die Digitalisierung ermöglicht auch eine bessere Standardisierung der externen Schnittstellen zu Lieferanten entlang der Wertkette im traditionellen Geschäft. Dies hat Einfluss auf die Transaktionskosten der externen Koordination, ${ }^{17}$ d. h. die Kosten der Transaktionen mit Lieferanten im Markt (,buy“) im traditionellen Geschäft. Auch diese sind Kosten der Anbahnung, Vereinbarung und Abwicklung von Transaktionen im traditionellen Geschäft mit Lieferanten sowie nachträglicher Kontrollen und Anpassungen.

Die Transaktionskosten (G) einer Eigenfertigung im Unternehmen (,make“) im Vergleich zum Fremdbezug bei Lieferanten (,buy“) am Markt ( $\Delta \mathrm{G})$ steigen mit höherer Faktorspezifität deutlich an. Sie müssen allerdings bei der Entscheidung über die externe Koordination den Produktionskosten (P) gegenübergestellt werden (Abb. 2.4). Grundsätzlich haben effizient spezialisierte Lieferanten im Markt einen Produktionskostenvorteil gegenüber der Eigenfertigung im Unternehmen $(\Delta \mathrm{P}>0)$. Dieser Vorteil nimmt allerdings mit steigender Spezifität der gelieferten Teile ab, weil der Lieferant durch Anpassungen Größen- und Erfahrungsvorteile verliert. ${ }^{18}$ Werden Transaktionsund Produktionskostenunterschiede gemeinsam betrachtet, dann bestehen bis zu einer Faktorspezifität in Höhe von $\mathrm{k}^{*}$ Anreize für die Auslagerung auf spezialisierte Lieferanten, weil die Spezialisierungsvorteile der Lieferanten selbst Transaktionskostennachteile mehr als kompensieren. Bei einer Faktorspezifität größer als $\mathrm{k}^{*}$ überwiegen dagegen die Transaktionskostennachteile zunehmend 
die Produktionskostenvorteile bei externer Koordination, weshalb sich Unternehmen für Eigenfertigung entscheiden werden (vgl. ebenfalls Abb. 2.4).

Durch die Digitalisierung können nicht nur Vor- und Zwischenprodukte schneller und einfacher von Lieferanten am Markt beschafft, sondern auch Informationen mit ihnen ausgetauscht werden. So lassen sich die Transaktionskosten der externen Koordination ${ }^{19}$ im traditionellen Geschäft $\left(\Delta G^{\prime}\right)$ reduzieren. Damit wird es einfacher, Produktionskostenvorteile spezialisierter Lieferanten durch Auslagerung zu nutzen (Verschiebung der kritischen Faktorspezifität, ab der Eigenfertigung in der Hierarchie erfolgt, von $\mathrm{k}^{*} \mathrm{zu} \mathrm{k}^{* *}, \mathrm{Abb}$. 2.4). Die Kosteneinsparungen können ebenfalls entweder für zusätzliche Wertschöpfung (Erhöhung der Variantenvielfalt) verwendet oder als verringerte Kosten einbehalten werden.

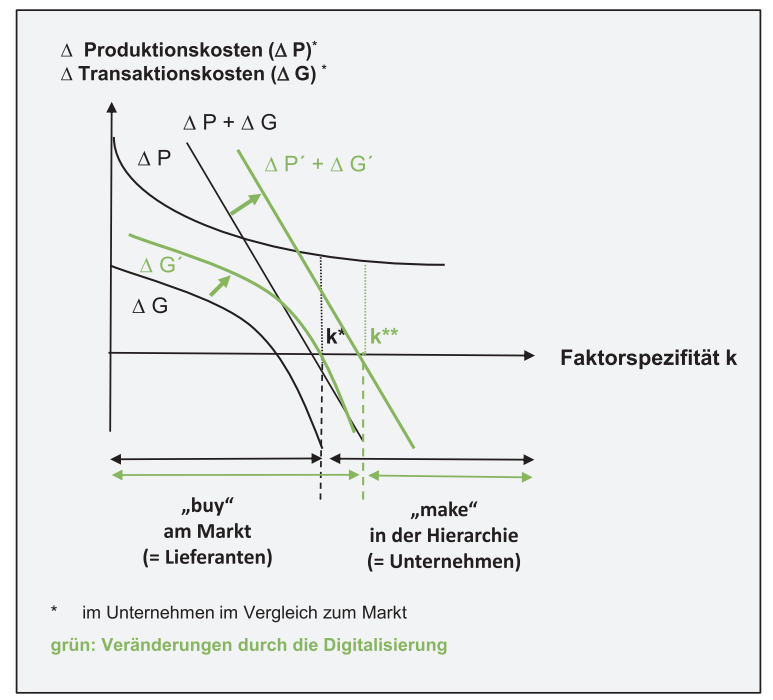

Abb. 2.4 Verringerung der Transaktionskosten der externen Koordination von Lieferanten im traditionellen Geschäft durch die Digitalisierung. (Quelle: nach Williamson 1975) 
(3) Verringerung der Transaktionskosten der externen Koordination von Kooperationspartnern im traditionellen Geschäft durch die Digitalisierung

Die bessere Standardisierung der externen Schnittstellen zu Kooperationspartnern entlang der Wertkette im traditionellen Geschäft durch die Digitalisierung erleichtert auch Kooperationen in Bereichen der Wertschöpfung, die nicht ausgelagert werden sollen. Dies hat ebenfalls Einfluss auf Transaktionskosten der externen Koordination, ${ }^{20}$ hier von Kooperationspartnern, im traditionellen Geschäft. Der Zusammenschluss von verschiedenen Unternehmen in inter-organisationalen Netzwerken stellt eine hybride Koordinationsform zwischen einer unternehmensinternen Wertschöpfung (Hierarchie) und einer Auslagerung auf Zulieferer (Markt) dar (vgl. Abb. 2.5). Partner inter-organisationaler Netzwerke können sich z. B. die Kosten der Produktion bei mittlerer Spezifität und Unsicherheit ${ }^{21}$ teilen.

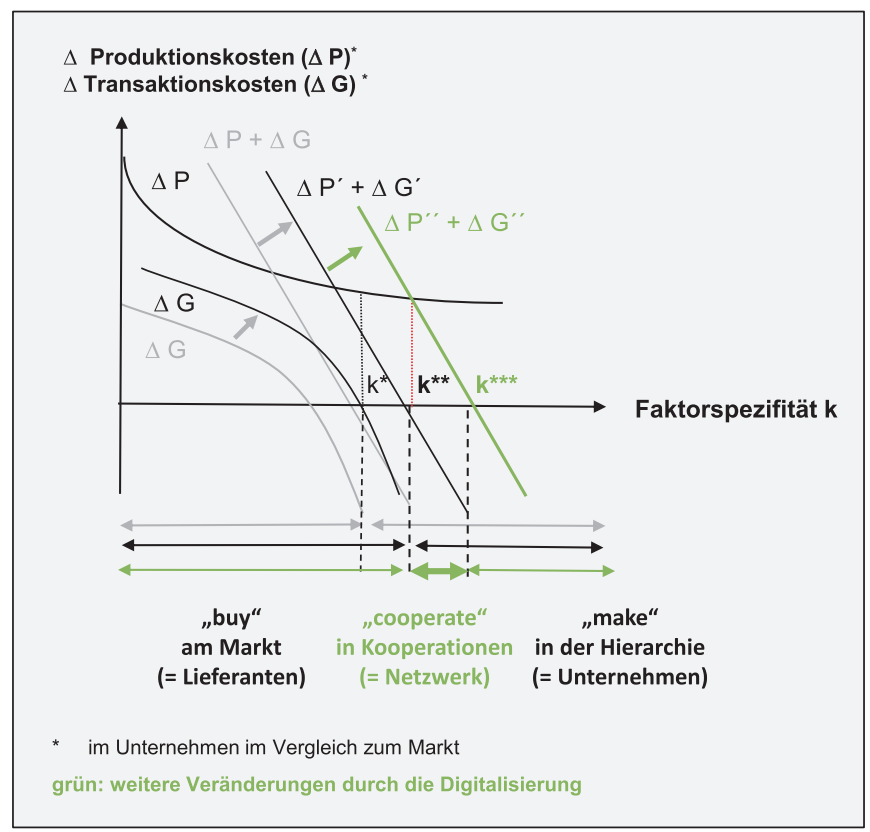

Abb. 2.5 Verringerung der Transaktionskosten der externen Koordination von Kooperationspartnern im traditionellen Geschäft durch die Digitalisierung. (Quelle: eigene Erweiterung von Abb. 2.4 mit Picot u. a. 2015). 
Diese Transaktionskosten der externen Koordination von Kooperationspartnern sinken durch die Digitalisierung $\left(\Delta \mathrm{G}^{\prime \prime}\right)$, weil die Koordination einer Partnerschaft bzw. eines Netzwerks im traditionellen Geschäft bei digitaler Planung, Steuerung und Datenverarbeitung reibungsloser abläuft. Damit wird es einfacher, Produktionskostenvorteile in Partnerschaften bzw. Netzwerken zu nutzen. Durch gemeinsame Wertschöpfung lassen sich zudem Synergien in Elementen der Wertkette, die z. B. aus Kompetenzüberlegungen nicht aufgegeben werden sollen, verwirklichen. Die Kosteneinsparungen können sie wiederum entweder für zusätzliche Wertschöpfung (Erhöhung der Variantenvielfalt) verwenden oder als verringerte Kosten einbehalten. Die kritische Faktorspezifität für eine interne Produktion (,make“) steigt in Abb. 2.5 gegenüber Abb. 2.4 von k** auf $\mathrm{k}^{* * *}$.

Die in den Abb. 2.3 bis 2.5 gezeigten Effekte einer Verringerung der (internen und externen) Transaktionskosten sind additiv - die Stärke der Nutzung hängt von den spezifischen Bedingungen in einem Unternehmen ab.

\section{(4) Größere Handlungsspielräume im Wettbewerb bei Verringerung der Zielkonflikte zwischen Effizienz und Flexibilität bei sinkenden Trans- aktionskosten im traditionellen Geschäft durch die Digitalisierung}

Die Digitalisierung beeinflusst durch bessere Standardisierung unternehmensinterner und -externer Schnittstellen (Abb. 2.3 und 2.5) entlang der Wertkette im traditionellen Geschäft (Abb. 2.1) auch Zielkonflikte im Management.

Ein wesentlicher Zielkonflikt im Management besteht zwischen Effizienz und Flexibilität, vor allem bei den primären Aktivitäten der Wertkette im traditionellen Geschäft, die in einer restriktiven Interdependenzbeziehung stehen (Abb. 2.1). Er liegt darin begründet, dass Flexibilität z. B. durch Halten von Kapazitätsreserven und Ereignispuffern (,slack“-Potenzialen) Effizienzverluste mit sich bringt, weil sie effiziente Aktivitäten entsprechend der Minimalkostenkombination (ohne jegliche ,slack“-Potenziale) verhindert. ${ }^{22}$ Dieser Konflikt konnte durch Verwendung von Produktplattformen und Gleichteilen wie z. B. Modulbaukästen in der Autoindustrie etwas verringert werden, die Einsparpotenziale (bei der Produktion der Plattformen und Gleichteile) und gleichzeitig Differenzierungsmöglichkeiten in Marketing und Vertrieb ermöglichen und durch neue Kombination der Gleichteile auch Innovationspotenziale schaffen. Dies vergrößert den Handlungsspielraum im Wettbewerb (Abb. 2.6).

Durch Verringerung der restriktiven Interdependenzen und damit der in- und externen Transaktionskosten entlang der Wertkette im traditionellen Geschäft (Abb. 2.1) verbessert die Digitalisierung nochmals mehr die Möglichkeit, effizient und gleichzeitig flexibel zu sein. Sie schafft Unternehmen neue Handlungsspielräume und Freiheitsgrade für eine Ausdifferenzierung von Leistungen und Geschäftsmodellen sowie eine bessere Ausrichtung im Wettbewerb. ${ }^{23}$ 


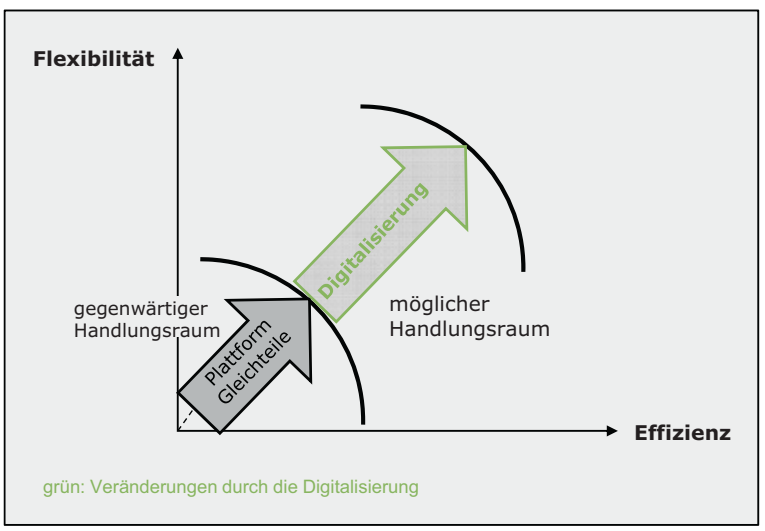

Abb. 2.6 Vergrößerung des Handlungsspielraums durch Digitalisierung. (Quelle: nach Proff 2019)

\subsection{Zusammenarbeit auf technischen Plattformen durch Digitalisierung als Voraussetzung für neue, innovative Kundenlösungen und Geschäftsmodelle}

Durch eine stärkere Verbindung der IT mit dem traditionellen Geschäft schafft die Digitalisierung zudem technische Plattformen als Schnittstellen zwischen Unternehmen, über die mehr als zwei Marktteilnehmer in neuartigen interorganisationalen Netzwerken interagieren sowie innovative Kundenlösungen und Geschäftsmodelle entwickeln können. Damit erhöht die Digitalisierung die Interaktion $^{24}$ in derartigen Netzwerken als Gemeinschaften verbundener Akteure, zwischen denen (Win-win-)Beziehungen bestehen. ${ }^{25}$

Solche Netzwerke können sehr unterschiedlich gestaltet sein: Sie können der gemeinsamen Entwicklung dienen wie z. B. die Entwicklungsplattform des Kartendienstes Here oder der gemeinsamen Marktbearbeitung wie die Mobilitätsangebote, die die Volkswagen We-Plattform bündelt. Sie können zudem von 
gleichberechtigten Unternehmen getrieben werden („,cooperation of equals“ wie z. B. Here) oder von einem dominanten Partner getrieben werden (wie z. B. Volkswagen We, vgl. den Kasten).

Wie kann ein Fahrzeug zu einem Plattform-Ecosystem werden? Mehrere Automobilunternehmen versuchen derNutzenversprechen ihres Fahrzeugs zu erweitern. Ein Beispiel, wie ein Fahrzeug zur Serviceplattform werden kann, das Volkswagen We-Ecosystem. Volkswagen wirbt damit, dass darin alle Angebote auf die spezifischen Kunden bedürfnisse ausgerichtet werden und der Kunde damit Teil des interaktiven Ecosystems ist. Durch die Vernetzung Fahrzeug und Kunde ist es möglich, digitale Services für den Kunden anzubieten und ihm somit einen entscheidenden Mehrwert zu bieten. Neben den hausinternen Volkswagen We-Diensten des Volkswagen Car-Net wie Security \& (automatischer Notruf), Guide \& Inform (Verkehrsinfo), e-Remote (Echtzeitinformationen für E-Modelle und Hybride) und App-Connect (Smartphone-Integration) können auch externe komplementäre Unternehmen Leistungen rund das Fahrzeug anbieten. So können Unternehmen über die Funktion We Deliver Hemden aus der Reinigung und Essen ins Fahrzeug liefern (Volkswagen We: The digital ecosystem 2019).

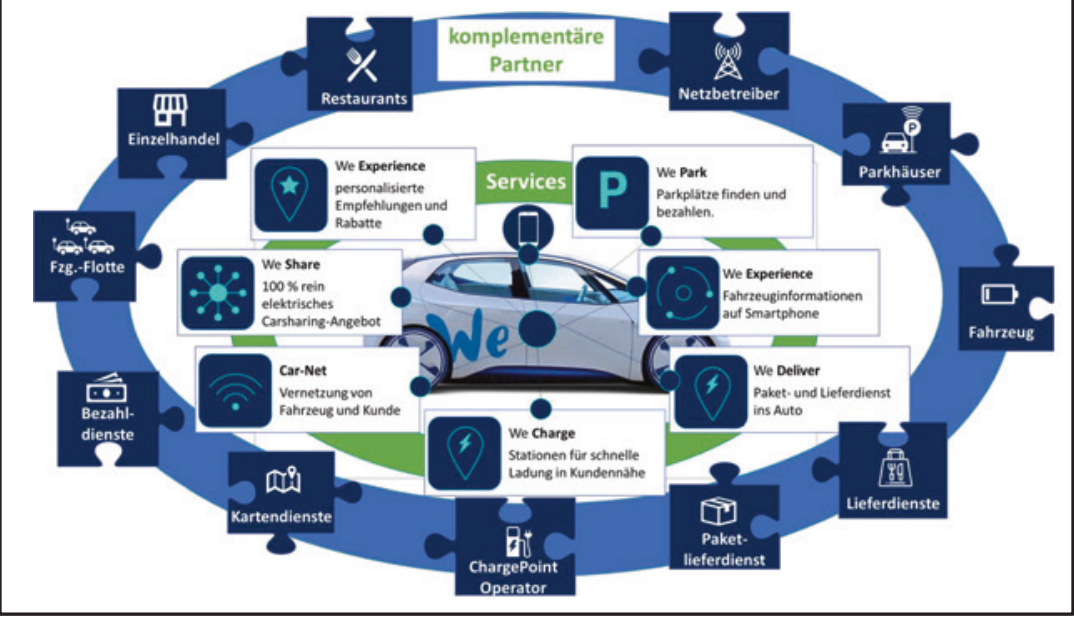

Voraussetzung für eine ökonomisch sinnvolle Interaktion über technische Plattformen ist Komplementarität durch gleichgerichtete Aktivitäten der Partner in solchen Netzwerken. ${ }^{26}$ Das lässt sich mit einem traditionellen mikroökonomischen Optimierungsmodell begründen. Bei der Zielfunktion handelt es sich um eine nicht-lineare und nicht-konvexe Gewinnfunktion $(\boldsymbol{\pi})$. Sie kann mehr als ein Optimum aufweisen (Abb. 2.7), was bei komplexen Entscheidungen z. B. in Unternehmensnetzwerken durchaus realistisch ist, weil oft mehrere Lösungen möglich sind. Solche Optimierungsprobleme lassen sich nicht mit den Standardmethoden des Operations Research (OR) lösen, sondern nur über supermodulare Funktionen, die es ermöglichen, aus der Vielzahl relativer Optima eine gewinnmaximale Zusammenführung der Wertschöpfungselemente der Partner abzuleiten. 


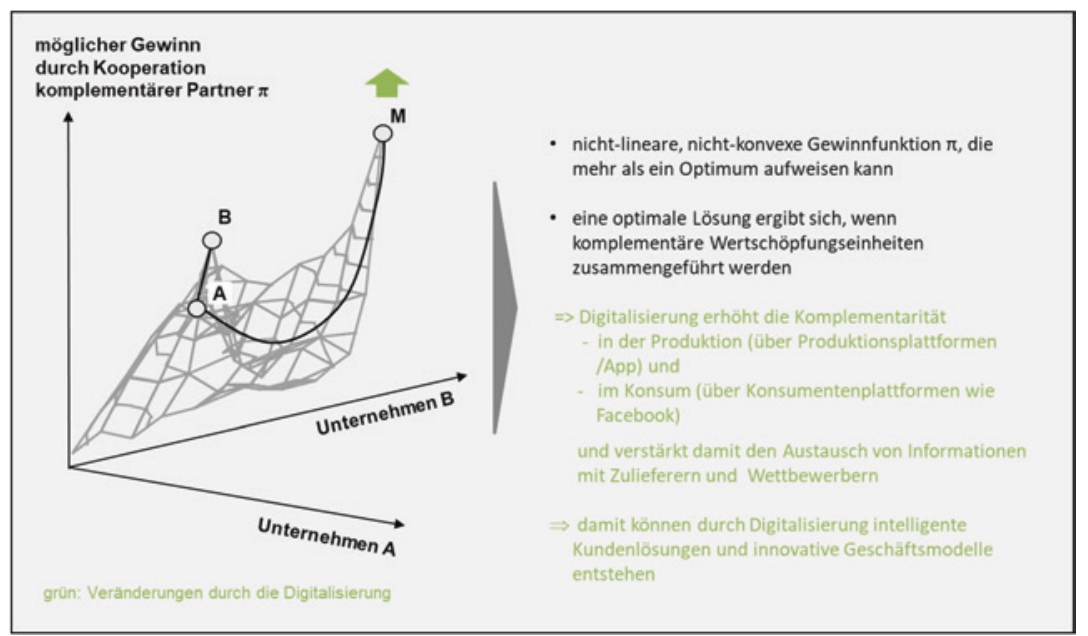

Abb. 2.7 Verstärkte Zusammenarbeit komplementärer Partner in strukturellen Ecosystemen durch die Digitalisierung. (Quelle: nach Milgrom, Roberts 1990, 1992 und Jacobides u. a. 2018)

Gemäß der Theorie der zwei- oder mehrseitigen Märkte (,two-(or multi-) sided markets“) lassen sich durch dichte Interaktion komplementärer Marktpartner, die jeweils eigene Interessen vertreten und auf Plattformen wechselseitig interagieren, ${ }^{27}$ in intra-organisationalen Netzwerken positive Netzwerkeffekte schaffen, indem der Gesamtnutzen im Netzwerk größer ist als die Summe der Einzelnutzen. ${ }^{28}$ Diese ökonomischen Vorteile werden statt als Komplementarität ${ }^{29}$ auch als Netzwerksynergien ${ }^{30}$ bezeichnet. Sie werden durch Wertschaffung bei Kooperation der Netzwerkpartner (,value co-creation“) ${ }^{31}$ und Wertaneignung im Wettbewerb (,value capture“"32) beschrieben und mit der kooperativen und nichtkooperativen Spieltheorie erklärt. Komplementaritäten steigen mit steigender Anzahl der Plattformnutzer.

Nach der Intensität der Beziehungen kann zwischen traditionellen losen Netzwerken und strukturellen Ecosystemen unterschieden werden. Letztere schaffen eine stärkere Interaktion einer Gruppe durch Abstimmung (,,alignment"*) in einem Netzwerk. ${ }^{33}$ Dies begründen ältere verhaltenswissenschaftliche Erklärungen des ,,alignment" in der Organisationsstruktur bzw. in Netzwerken, wie z. B. der Ansatz der Clanorganisation. ${ }^{34}$ In einem solchen strukturellen Ecosystem wird ein bestimmter 
Kreis an Partnern in einer multilateralen Beziehung an einem unternehmensübergreifenden Wertschöpfungsnetzwerk verbindlich ausgerichtet, um ein übergeordnetes Nutzenversprechen zu schaffen. ${ }^{35}$ Anders als traditionelle Netzwerke, die bei den Akteuren beginnen, richten sich Ecosysteme als Struktur auf Aktivitäten aus und geben ein übergeordnetes Nutzenversprechen ab. Dabei ist zumindest für den Koordinator einer solchen Lösung das Ecosystem die einzige Koordinationsform. Die Partnerunternehmen agieren gleichzeitig auch als eigenständige Unternehmen (Hierarchie) und geben in einem Netzwerk nicht alle eigenen Kompetenzen preis.

Die Digitalisierung erhöht Komplementaritäten bzw. Netzwerksynergien in der Produktion und im Konsum und verstärkt damit den Austausch von Informationen mit Zulieferern und Wettbewerbern, die in einem strukturellen Ecosystem verbunden sind (vgl. ebenfalls Abb. 2.7). In der Produktion werden solche Komplementaritäten über eine entsprechende Plattform geschaffen, z. B. wenn auf einem Betriebssystem mehrere Apps angeboten werden, im Konsum entstehen sie über eine Konsumentenplattform wie Facebook. ${ }^{36}$ Damit können durch die Digitalisierung intelligente Kundenlösungen und innovative Geschäftsmodelle entstehen und Gewinnmöglichkeiten geschaffen werden, die ein Marktteilnehmer alleine oft nicht erbringen kann (vgl auch das folgende Video 2).

Video 2: Wertschaffung und -verteilung in den digitalen Ecosystemen (https://doi. org/10.1007/000-0sw)

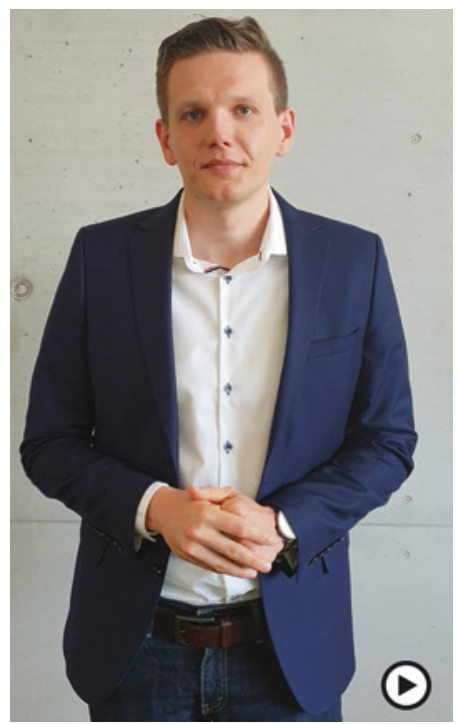


In diesem Kapitel wurde gezeigt, dass die Digitalisierung dem Management Handlungsspielräume schafft und Gewinnpotenziale ermöglicht. Da das allerdings auch für die Wettbewerber gilt, sind zur Nutzung der Chancen der Digitalisierung konsequente Handlungen erforderlich. In den nächsten Kapiteln wird nun der Frage nachgegangen, wo die Vorteile im Management ansetzen (Kap. 3) und wie die Chancen der Digitalisierung durch Veränderungsfähigkeiten genutzt werden können (Kap. 4).

\section{Anmerkungen zu Kapitel 2}

1. Vgl. zu diesem Kapitel auch Proff (2002 und 2019).

2. Vgl. Williamson (1975) oder Riordan, Williamson (1985).

3. Vgl. Porter (1985) bezogen auf Gluck (1980).

4. Vgl. z. B. Frese (2000, 2. Teil), aber auch Thompson (1967, Kap. 5) und darauf bezogen Proff (2002, S. 93).

5. Frese (2000, S. 59).

6. Vgl. Thompson (1967, S. 54) oder Frese (2000, S. 54).

7. Vgl. Porter (1985); Schweitzer (2000) und Betz (1999, S. 112).

8. Porter (1985).

9. Vgl. z. B. Cyert, March (1963, S. 117).

10. Frese (2000, S. 54).

11. Vgl. Hagiu, Wright (2015).

12. Dies umfasst z. B. auch einen durchgängigen Kundenauftragsprozess, der die Daten des Kundenauftrags sofort auch den Lieferanten zur Verfügung stellt. Die so gewonnenen Tage und oft Wochen reichen, um von einem spezialisierten Lieferanten quasi just-in-time Zulieferteile zu bekommen.

13. Vgl. Deloitte (2020a).

14. Vgl. Knobbe, Proff (2020).

15. Vgl. z. B. Picot u. a. (2015).

16. Vgl. Hagiu, Wright (2015).

17. Vgl. ebd.

18. Vgl. Grossman, Hart (1986).

19. Vgl. ebd.

20. Vgl. ebd.

21. Vgl. Picot u. a. (2015).

22. Vgl. z. B. Mette (1999, S. 144), aber auch Cyert, March (1963, S. 36-37).

23. Weil im internationalen Management ein Zielkonflikt zwischen einer länderübergreifenden, zentralen und damit effizienzorientierten Steuerung ausländischer Tochtergesellschaften und einer flexibilitätsorientierten, dezentralen und damit länderspezifischen Steuerung besteht, den eine 
Digitalisierung verringern kann, bietet diese auch Ansatzpunkte für eine Neuausrichtung der Steuerung ausländischer Tochtergesellschaften (vgl. Proff 2019).

24. Vgl. z. B. Gawer (2014).

25. Vgl. Adner (2017) und dazu auch Szybisty (2020).

26. Vgl. Milgrom, Roberts $(1990,1992)$ und darauf bezogen Proff, Proff (2013, Abschn. 7.3).

27. Vgl. z. B. Haigu, Wright (2015, S. 163).

28. Vgl. z. B. Gawer (2014).

29. Vgl. Jacobides u. a. (2018) und Hannah, Eisenhardt (2018).

30. Vgl. z. B. Huth (2015).

31. Z.B. Vargo, Lusch (2004 und 2008).

32. Vgl. z. B. Gans, Ryall (2017).

33. Vgl. Adner (2017) und Szybisty (2020).

34. Vgl. z. B. Schoppe u. a. (1995, S. 246-261) oder Schreyögg (2008, S. 318329).

35. Vgl. ebd. (S. 42).

36. Vgl. Jacobides u. a. (2018).

Open Access Dieses Kapitel wird unter der Creative Commons Namensnennung 4.0 International Lizenz (http://creativecommons.org/licenses/by/4.0/deed.de) veröffentlicht, welche die Nutzung, Vervielfältigung, Bearbeitung, Verbreitung und Wiedergabe in jeglichem Medium und Format erlaubt, sofern Sie den/die ursprünglichen Autor(en) und die Quelle ordnungsgemäß nennen, einen Link zur Creative Commons Lizenz beifügen und angeben, ob Änderungen vorgenommen wurden.

Die in diesem Kapitel enthaltenen Bilder und sonstiges Drittmaterial unterliegen ebenfalls der genannten Creative Commons Lizenz, sofern sich aus der Abbildungslegende nichts anderes ergibt. Sofern das betreffende Material nicht unter der genannten Creative Commons Lizenz steht und die betreffende Handlung nicht nach gesetzlichen Vorschriften erlaubt ist, ist für die oben aufgeführten Weiterverwendungen des Materials die Einwilligung des jeweiligen Rechteinhabers einzuholen.

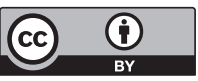

